\title{
Tumors Originating from Fat Tissue in the Head and Neck Area: A Review of 40 Cases
}

\author{
Atsushi Ochiai* \\ Department of Otolaryngology-Head and Neck Surgery, School of Medicine, Kitasato University, Japan \\ *Corresponding author: Atsushi Ochiai, Department of Otolaryngology-Head and Neck Surgery, School of Medicine, \\ Kitasato University, 1-15-1 Kitasato, Minami-ku, Sagamihara-shi, Kanagawa, 252-0374, Japan
}

\section{ARTICLE INFO}

Received: 幽 January 17, 2022

Published: January 26, 2022

Citation: Atsushi Ochiai. Tumors Originating from Fat Tissue in the Head and Neck Area: A Review of 40 Cases. Biomed J Sci \& Tech Res 41(2)-2022. BJSTR. MS.ID.006589.

Keywords: Lipoma; Age; Sex; Chief complaints; Growth style; Size; Primary lesion; Treatment; Complications; Prognosis

\section{ABSTRACT}

Objectives: To clarify the features of lipomas that had been operated on in Kitasato University Hospital (KUH) and diagnosed by reviewing a series of cases.

Method: All patients having undergone tumor removal between January 2000 and December 2021. Patients were identified through pathology department records.

Results: Ages ranged from 2 to 80 years, with an average age of 47.1 years. Lipomas were benign in 38 cases and malignant in 2 cases. All 38 lipomas required only surgery. In cases of lipoma, 3 cases developed complications after surgery. In the cases of lipoma, although postoperative results were varied and changing, there were no recurrences.

Conclusion: Clinical files were reviewed for 40 patients. Lipomas were benign in 38 cases and malignant in 2 cases. Complications after surgery were seen in 3 cases. Although treatment requires total removal, in some cases require preoperative or postoperative chemotherapy and radiotherapy.

Abbreviations: KUH: Kitasato University Hospital; RLN: Recurrent Laryngeal Nerve; CT: Computed Tomography; MRI: Magnetic Resonance Imaging

\section{Introduction}

Lipomas, including spindle cell lipomas, pleomorphic lipomas, angiolipomas, lipoblastomas, and so on are benign tumors originating from fat tissue. Lipomas are the most frequently occurring benign tumors, and most lipomas arise subcutaneously. Liposarcoma is a malignant tumor consisting of a lipoblast. But, the degree of differentiation among all of these tumors are various, and the growth speed, metastasis, and prognosis of these tumors differ substantially by histological type. I therefore undertook this study to clarify the features of lipomas that had been operated on in Kitasato University Hospital (KUH) and diagnosed by reviewing a series of cases.

\section{Materials and Methods}

A. Ethical Consideration: patients included in this study were not subject to any harm. I ensured all patients had their privacy protected by ensuring anonymity of the data collected.

B. Study Design: A retrospective study of all patients who underwent tumor removal.

C. Setting: All patients having undergone tumor removal at Department of Otolaryngology-Head and Neck Surgery, KUH between January 2000 and December 2021. Patients were identified through pathology department records. 
D. Participants: In each case, I recorded age, sex, pathological results, chief complaints, growth style, size, time until the first visit, primary lesion, treatment, complications and prognosis.

\section{Results}

\section{Age and Sex (Figure 1)}

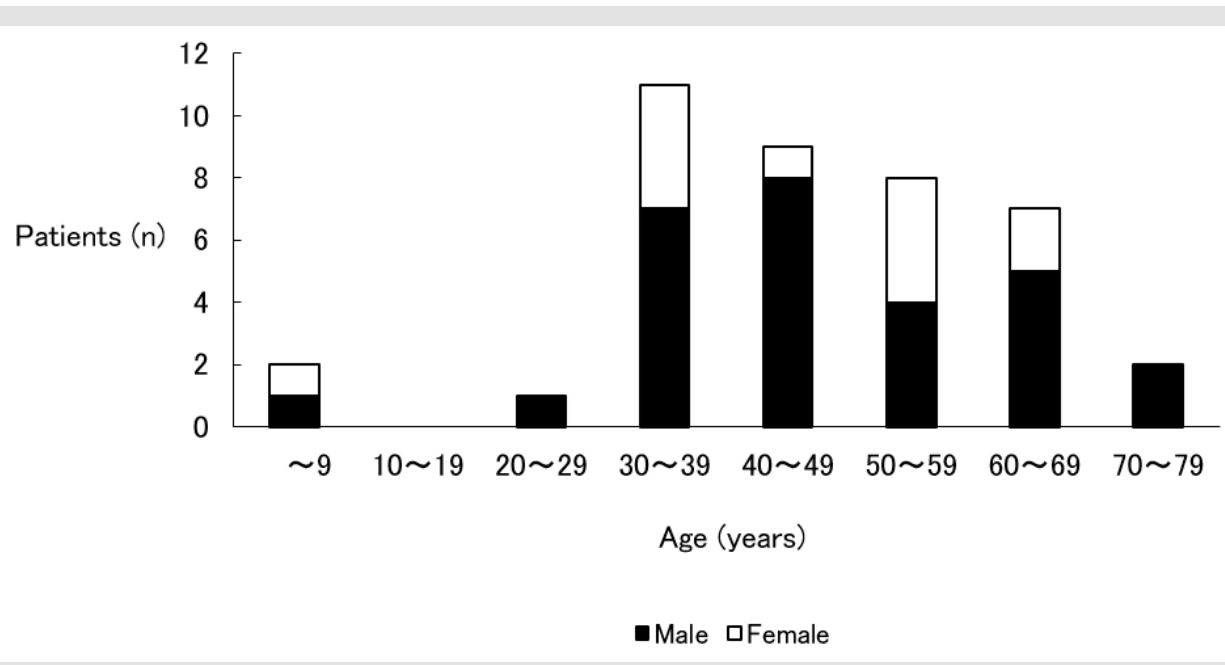

Figure 1: Age and Sex.

Note: Ages ranged from 2 to 80 years, and it was most common in the third decade of life. Sex difference was that male predominated.

Ages ranged from 2 to 80 years, with an average age of 47.1 years. Tumor removal was most common in the third decade of life. Sex difference: male (28 cases) and female (12 cases), so male predominated.

\section{Pathological Results}

Lipomas were benign in 38 cases (95\%) and malignant in 2 cases (5\%). One liposarcoma was a recurrent case; the other was a fresh case.

\section{Chief Complaints}

Swelling of the primary lesion without pain was observed in 37 cases (92.5\%). Swelling of the primary lesion with pain was observed in 1 case (2.5\%); this was a fresh case of liposarcoma. The remaining 2 cases (5 \%) were asymptomatic and were accidentally found by image examination or by examination in another department.

\section{Growth Style}

Growth style was either unchanged (16 cases; $40 \%$ ) or slow (24 cases; $60 \%$ ). Two cases of liposarcoma were slow.

\section{Size (Figure 2)}

Lipoma size classes (along its major axis) were counted, and the following results were obtained: smaller than $1 \mathrm{~cm}$ (1 case; 2.5
\%); 1 to $5 \mathrm{~cm}$ (24 cases; $60.0 \%$ ), the most common and accounting for more than half of all cases; 5 to $10 \mathrm{~cm}$ (10 cases; $25.0 \%$ ); and larger than $10 \mathrm{~cm}$ (5 cases; $12.5 \%$ ). The size of the recurrent case of liposarcoma was $6 \mathrm{~cm}$, and the size of the fresh case was $11 \mathrm{~cm}$.

\section{Time Until the First Visit (Figure 3)}

A count of the times until the first visit to KUH was made, and the following results were obtained: within 1 week ( 4 cases; 10.0 \%); 1 week to 1 month ( 1 case; $2.5 \%$ ); 1 month to 6 months (14 cases; $35.0 \%$ ), the most common; 6 months to 1 year ( 2 cases; 5.0 $\%$ ); 1 year to 5 years (12 cases; $30.0 \%$ ); and over 5 years ( 7 cases; $17.5 \%)$. There were 19 cases ( $47.5 \%$ ) that had been left for more than 1 year. In the recurrent case of liposarcoma, 6 days elapsed, and in the fresh case, 2 months elapsed.

\section{Primary lesion (Figure 4)}

The neck was the most common with 18 cases (45\%) and was followed by the submandibular (10 cases; $25 \%$ ), the parotid gland ( 5 cases; $12.5 \%$ ), the nasopharynx ( 2 cases; $5 \%$ ), the face ( 2 cases; $5 \%$ ), the opisthotic (1 case; $2.5 \%$ ), the tongue (1 case; $2.5 \%$ ), and the mentum (1 case; $2.5 \%$ ). Laterality was right side in 27 cases $(67.5 \%)$ and left side in 10 cases (25\%), so the right side predominated. The remaining 3 cases $(7.5 \%)$ occurred at the nasopharynx and the mentum and were middle. Both cases of liposarcoma occurred at the right neck. 


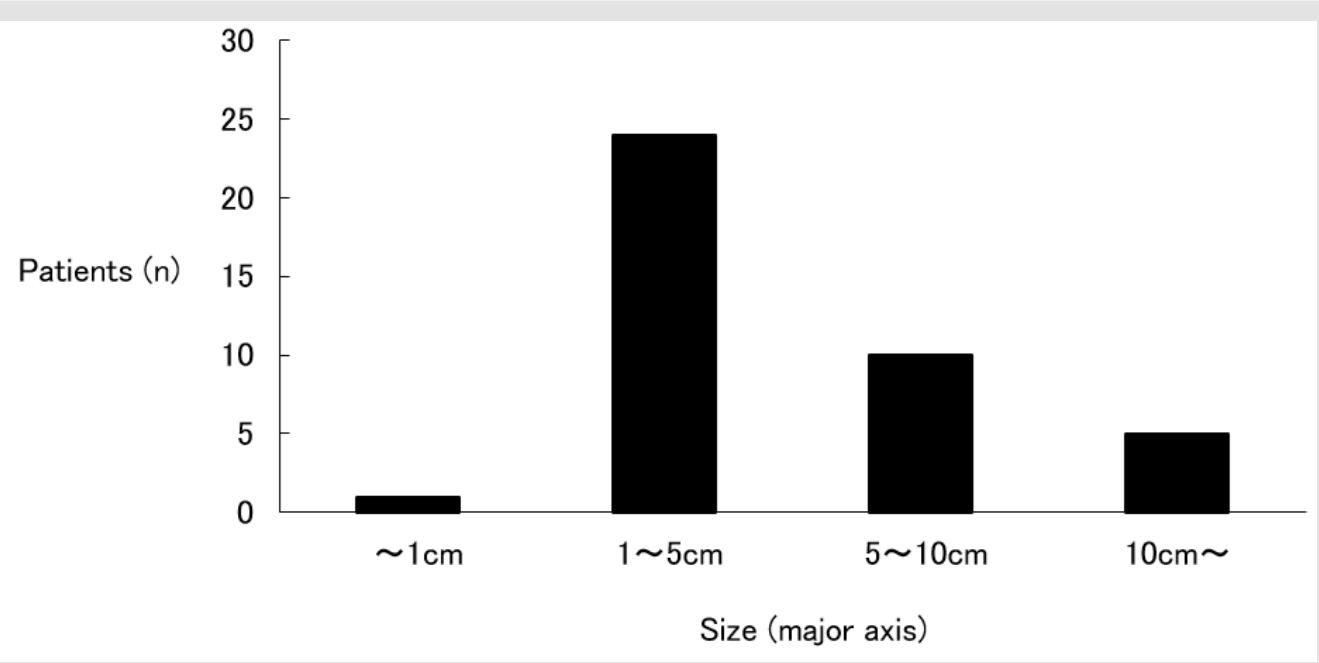

Figure 2: Size.

Note: 1 to $5 \mathrm{~cm}$ was the most common and accounting for more than half of all cases.

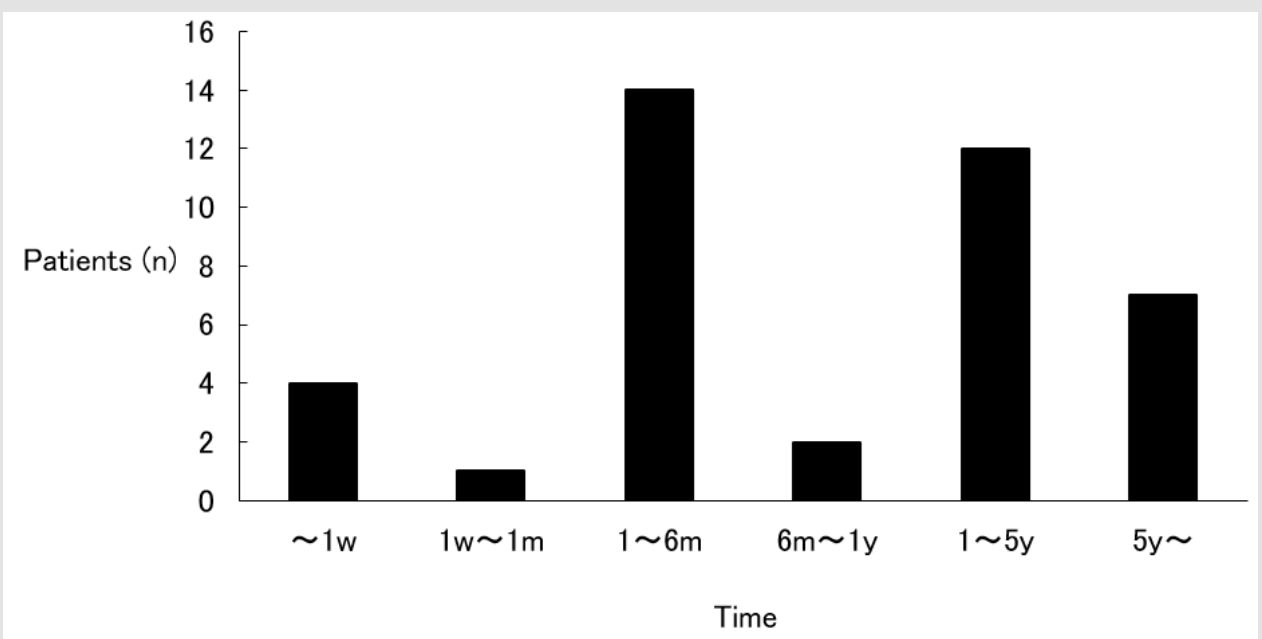

Figure 3: Time until the first visit

Note: Although 1 month to 6 months was the most common, there were 19 cases that had been left for more than 1 year. w: week, m: month(s), y: year(s)

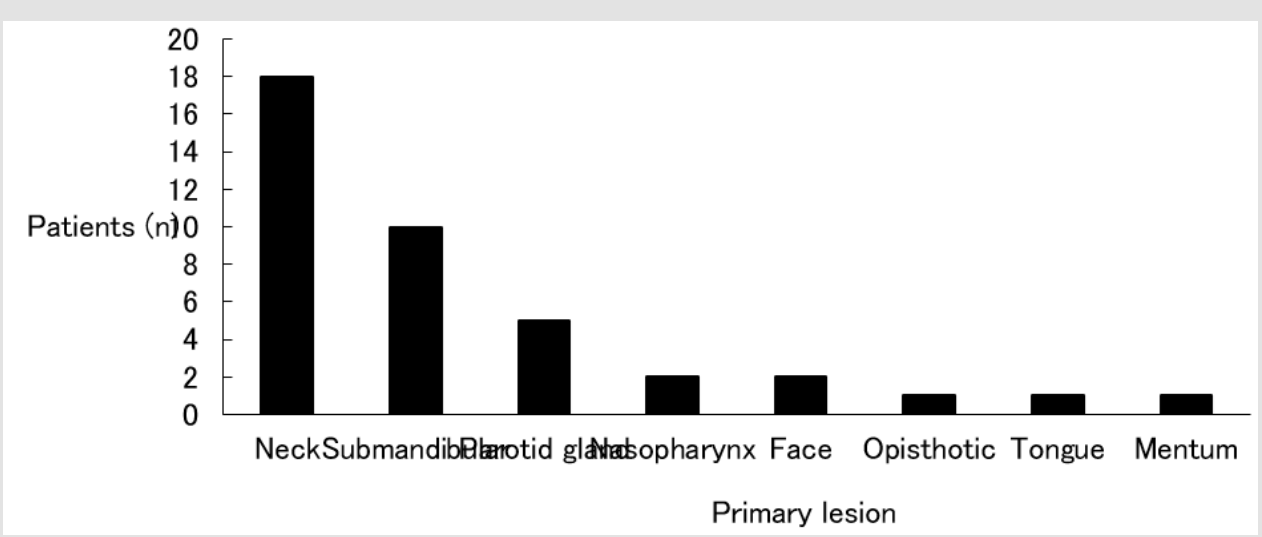

Figure 4: Primary lesion.

Note: The neck was the most common with 18 cases. 


\section{Treatment}

All 38 lipomas required only surgery. One of 2 liposarcomas was a recurrent case after surgery at another hospital. Dyspnea had occurred 1 week before the scheduled surgery date at $\mathrm{KUH}$, and an emergency tracheostomy was performed. Surgery was still performed according to schedule 1 week later. In the fresh case of liposarcoma, because internal carotid artery was coarctated by the tumor, it was determined that surgery would be impossible. After a radiotherapy dose of 60 gray, insertion of superficial temporal artery catheter, and administration of $252.5 \mathrm{mg}$ CDDP from the catheter, the tumor reduced. Therefore, surgery would be possible and was performed.

\section{Complications}

In cases of lipoma, 3 cases developed complications after surgery. One hemorrhaged after surgery and required a tracheostomy; another resulted in transient palsy of the marginal branch of the facial nerve; and the last resulted in transient palsy of the marginal branch of the facial nerve, fistulation, and an abscess at the wound.

In fresh case of liposarcoma, the recurrent laryngeal nerve (RLN) was involved in the tumor. Therefore, the RLN had to be cut and an RLN palsy occurred.

\section{Prognosis}

In the cases of lipoma, although postoperative results were varied and changing, there were no recurrences. In the cases of liposarcoma, the recurrent case died by another disease, and the fresh case is unknown because a change of address occurred. But, there were no recurrences or metastases during the follow-up period.

\section{Discussion}

Lipoma is a benign tumor that is composed of mature fat cells that grow relatively slowly. Pathologically, it is composed of crescentshaped and unevenly distributed nuclei, and round or oval cells that have monosegmental vacuolar cell bodies. It forms various sized lobules and is separated by narrow connective tissue. Lipoma is a soft globular or lobulated tumor by palpation, and there is almost no pain. Therefore, if there are no cosmetic problems or difficulties in daily life, patients tend to live for a long time. In this study, there were 19 cases ( $48.7 \%$ ) that lived for more than 1 year. Although treatment requires total removal, in some cases that lipoma grows not only subcutaneously but also inside requires partial removal [1]. The growth styles of all cases were unchanged and slow, and there were no complaints of pain in this study. Further, all patients could be totally removed, and there were no recurrences.

Although liposarcoma is one of the frequently encountered soft tissue tumors, liposarcoma in the head and neck area is rare (5.67
\%) [2]. Pathologically, liposarcoma is composed of lipoblasts, and there are various differentiated types ranging from well to poor. Growth speed, metastasis, and the survival rate after 5 years varies markedly by differentiated type. Round cell types and pleomorphic types cause metastasis in the early stages, and prognosis is much worse. Differentiated type and mucous type have a low rate of metastasis, and prognosis is much better. Because the latter is greater than the former, liposarcoma is considered to be a tumor with a relatively better prognosis [3]. There were atypical nuclei abounding in chromatin and both large and small vacuolar and lipoblast cells here and there in the recurrent case. As to treatment, there is general removal and preoperative or postoperative chemotherapy and radiotherapy. Postoperative radiotherapy was not added in the recurrent case because the patient was in advanced age. Preoperative radiotherapy and chemotherapy were both performed in the fresh case. The recurrent case was pleomorphic type, and the fresh case was mucous type. Both cases did not cause recurrence and metastasis during the postoperative follow-up period.

Although differential diagnosis between benign and malignant tumors is based on biopsy, it is said that 10 to $15 \%$ of malignant tumors have local pain or heat, clinically. Although the details of recurrent case were unknown, the fresh case did complain of pain. Further, although 2 liposarcoma cases did not perform computed tomography (CT) or magnetic resonance imaging (MRI) because they occurred in the early 1970's, Ito, et al. [4] reported that both CT and MRI show lipoma as an almost isolated signal in subcutaneous fat. The margins are clear, and except for well-differentiated types, liposarcomas differ from fat and contain the part of CT is high density, T1 of MRI is low intensity and T2 of MRI is high intensity. Therefore, differential diagnosis between liposarcoma and lipoma is possible, but differential diagnosis between well-differentiated types of liposarcoma and lipoma is difficult.

\section{Conclusion}

Clinical files were reviewed for 40 patients who had been operated on and diagnosed as lipoma in the head and neck area. Ages ranged from 2 to 80 years, and the majority of patients were male. Lipomas were benign in 38 cases and malignant in 2 cases. Complications after surgery were seen in 3 cases. Although treatment requires total removal, in some cases require preoperative or postoperative chemotherapy and radiotherapy.

\section{Financial Support}

This research received no specific grant from any funding agency, commercial or not-for-profit sectors.

\section{Conflict of Interest}

None. 


\section{References}

1. Oda A, Yoshihara T, Ishii T (1994) A clinical evaluation of lipoma, lipomatosis and liposarcoma in the head and neck region. Otologia Fukuoka.

2. Enzinger FM, Weiss SW (1995) Soft tissue tumors ( $3^{\text {rd }}$ Edn.)., St. Louis: CV Mosby.

ISSN: 2574-1241

DOI: 10.26717/BJSTR.2022.41.006589

Atsushi Ochiai. Biomed J Sci \& Tech Res

(C) (P) This work is licensed under Creative

Submission Link: https://biomedres.us/submit-manuscript.php
3. Omukai T, Ishida T, Takami M, Matsuda M, Terakawa F (1989) Postoperative chemotherapy for liposarcoma. Cent Jpn J Orthop Traumat.

4. Ito T, Nakanishi K, Sugiura E, Sato K, Miura T (1992) Utility of MR imaging and CT for lipomatous tumors. Cent. Jpn J Orthop Traumat.

$\begin{array}{ll}\text { BIOMEDICAL } & \text { Assets of Publishing with us } \\ \text { RESEARCHES } & \text { - Global archiving of articles } \\ \text { - Immediate, unrestricted online access }\end{array}$

